\title{
A novel image fusion algorithm based on 2D scale-mixing complex wavelet transform and Bayesian MAP estimation for multimodal medical images
}

\author{
Abdallah Bengueddoudj**, Zoubeida Messali* and Volodymyr Mosorov ${ }^{\dagger}$ \\ *Department of Electrical Engineering, University of Bordj \\ Bou Arreridj, 34030 El Anasser, Algeria \\ ${ }^{\dagger}$ Institute of Applied Computer Science, Lodz University of Technology \\ Stefanowskiego 18/22, 90-924 Lodz, Poland \\ tbeng.abdallah@hotmail.com
}

Received 14 April 2016

Accepted 1 November 2016

Published 30 December 2016

\begin{abstract}
In this paper, we propose a new image fusion algorithm based on two-dimensional Scale-Mixing Complex Wavelet Transform (2D-SMCWT). The fusion of the detail 2D-SMCWT coefficients is performed via a Bayesian Maximum a Posteriori (MAP) approach by considering a trivariate statistical model for the local neighboring of 2D-SMCWT coefficients. For the approximation coefficients, a new fusion rule based on the Principal Component Analysis (PCA) is applied. We conduct several experiments using three different groups of multimodal medical images to evaluate the performance of the proposed method. The obtained results prove the superiority of the proposed method over the state of the art fusion methods in terms of visual quality and several commonly used metrics. Robustness of the proposed method is further tested against different types of noise. The plots of fusion metrics establish the accuracy of the proposed fusion method.
\end{abstract}

Keywords: Medical imaging; multimodal medical image fusion; scale-mixing complex wavelet transform; MAP Bayes estimation; principal component analysis.

\section{Introduction}

Medical image fusion is increasingly used in diagnostics process due to the growing availability of medical imaging modalities. The different types of these modalities such as Magnetic Resonance Image
(MRI), Computed Tomography (CT), Positron Emission Tomography (PET) and Single Photon Emission Tomography (SPECT) offer complementary information about the human body. For example, MR images are particularly suitable for

\$Corresponding author.

This is an Open Access article published by World Scientific Publishing Company. It is distributed under the terms of the Creative Commons Attribution 4.0 (CC-BY) License. Further distribution of this work is permitted, provided the original work is properly cited. 
examining soft tissues of the body while CT images deliver more information about bones and hard tissues. Similarly, T1-MR image provides detailed information about soft tissues anatomy and fat whereas T2-MR image gives information about fluids and abnormal tissues such as tumours and inflammation. In clinical situations, the radiologists need information from multiple imaging modalities since a single imaging modality may not provide enough information. Therefore, image fusion is required to combine all relevant information from the different input images into a single fused image. Actually, dual modality imaging systems such as $\mathrm{MR} / \mathrm{SPECT}$ and $\mathrm{MR} / \mathrm{PET}^{1}$ are successfully applied to integrate both functional and anatomical information. SPECT $/ \mathrm{CT}^{2}$ and $\mathrm{MR} / \mathrm{CT}$ are used as well for the detection of malignant tumours and Alzheimer's disease, etc.

Several fusion techniques have been proposed in the literature and they can be classified into three different levels depending on the stage at which the fusion takes place, namely: pixel level, feature level and decision level. ${ }^{3}$ Fusion at pixel-level is performed directly on the values derived from the pixels of the input images on a pixel-by-pixel basis to generate a single fused image. Hence, pixel-based fusion methods become the most popular fusion techniques, while only few fusion algorithms use feature and decision level due to their complexity and difficulty of implementation.

The fusion process at any level should always preserve all pertinent information presented in the input images. Furthermore, it should be completed without introducing any artifacts, noise, unpredicted feature or loss information in the fused image. The existing fusion algorithms can be broadly grouped into three categories, ${ }^{4}$ viz. spatial domain techniques, transform domain techniques, and optimization approaches.

Spatial domain techniques such as averaging and weighted averaging are the simplest way for image fusion. However, these techniques extensively suffer from undesirable side effect such as contrast degradation. More robust methods that performed in the substitution domain such as the Principle Component Analysis (PCA) and Cross Bilateral Filter $(\mathrm{CBF})$ may provide better visual results and are easy to implement. Nevertheless, these methods do not provide any spectral information and produce spatial distortions in the fused image. Therefore, many algorithms based on multiresolution techniques have been developed to overcome these limitations. Pyramid and wavelet transforms are the most popular multiresolution approaches widely used in image fusion. The pyramid transform ${ }^{5}$ based fusion methods do not offer any directional information and suffer from blocking artifact in the region where the input images are different. To overcome these drawbacks, other pixel-level fusion methods based on wavelet transform have been developed. The wavelet transform ${ }^{6}$ offers good directional information and provides better representation in the decomposed components. Discrete Wavelet Transform (DWT) is one of the popular wavelet transforms used in image fusion. It has been shown that DWT-based fusion methods outperform the conventional image fusion methods based on pyramid transform in terms of a good localization in both the spatial and the frequency domains. One of the basic wavelet-based fusion methods was presented by Li et al. ${ }^{7}$; it includes a maximum selection rule to determine which of the wavelet coefficients are able to contain the most important information. The major drawback of this method is the use of the same fusion rule for combining both approximation coefficients and detail coefficients. Therefore, the choice of an appropriate fusion rule should consider the wavelet coefficient characteristics of each band. For instance, a DWT-based medical image fusion algorithm has been introduced by Chiorean and Vaida. ${ }^{8}$ It follows the maximum selection rule for coefficients combination in the high- frequency subbands and the simple averaging rule in the lowfrequency subbands. Recently, many methods have been introduced ${ }^{9}$ which involve wavelet-based image fusion that integrates the region-based scheme as a fusion rule. Lewis et al. ${ }^{10}$ have been proposed a joint segmentation of the input images into different regions. The image fusion task is carried out by a priority map, which is calculated using the variance, the energy or the entropy of the wavelet coefficients.

According to Liu and Moulin, ${ }^{11}$ a strong dependency exists between wavelet coefficients in both inter-subband and intra-subband. For this reason, many statistical models for image fusion have been developed. Furthermore, choosing an appropriate statistical model based on the properties of wavelet coefficients can improve the efficiency of the fusion process. The main difference between the statistical fusion methods is the prior distribution used to model the statistical behavior of the wavelet coefficients. In Ref. 12, a shrinkage approach has 
been integrated in the fusion scheme using anisotropic bivariate Laplacian probability density function (PDF). The fusion method proposed in Ref. 13, applies Hidden Markov Models (HMMs) and non-Gaussian prior function. Roy et al. ${ }^{14}$ propose a locally-adaptive algorithm where a multivariate Gaussian PDF was used. The method proposed in Ref. 15, uses a zero-mean bivariate Gaussian PDF over the local neighboring detail DWT coefficients of the input images. Moreover, Loza et al. ${ }^{16}$ have used Generalized Gaussian (GG) and alpha-stable prior to fuse noisy images.

The efficiency of the wavelet-based image fusion depends on the choice of both the wavelet and the applied fusion rules. The real valued wavelet transforms suffer from three major problems: shift sensitivity, lack of directionality and no phase information. ${ }^{17}$ Complex Wavelet Transforms (CWTs) has been proposed to overcome these limitations. CWT contains important information about the magnitude as well as the phase. ${ }^{18}$ According to Ref. 19, the magnitude of the complex wavelet coefficients provides information about smooth regions and edges while the phase indicates their locations. Furthermore, Dual Tree Complex Wavelet Transform (DTCWT), ${ }^{20}$ Nonsubsampled Contourlet Transform (NSCT) ${ }^{21}$ and Shearlet Transform (NSST) ${ }^{22}$ contain structural, edge and salient information of the input image. With this insight, many algorithms have been developed. ${ }^{23,24}$ However, these new schemes are time and memory consuming. Recently, a two-dimensional (2D) Scale-Mixing Complex Wavelet Transform (2DSMCWT) has been developed. ${ }^{25,26}$ The 2D-SMCWT preserves the energy that avoids the contrast distortions. Moreover, it has a better representation of the input images with several hierarchies of detail coefficients, and guarantees the orthogonality and the inverse transform can be performed in a straightforward manner. Therefore, a novel and efficient pixel-level image fusion algorithm based on 2D-SMCWT for multimodal medical images is proposed in this paper.

The innovative aspect of the present work is twofold: first, we use a trivariate Gaussian PDF as in Ref. 15 for the detail 2D-SMCWT coefficients while taking into account the mutual correlation between the input images and the fused image. A Bayesian MAP estimation technique has been used to obtain the detail 2D-SMCWT coefficients of the fused image. Second, we propose a new fusion rule based on the PCA to merge the approximation 2D-SMCWT coefficients. To highlight the advantage of the proposed method, a quantitative and qualitative comparative study is established with various well-known fusion methods. The remainder of this paper is organized as follows: Section 2 includes a brief review and preliminaries of the 2DSMCWT. Section 3 explains the proposed image fusion method. Experimental results and performance evaluations are presented in Sec. 4. Finally, Sec. 5 provides conclusions of our work.

\section{The 2D Scale-Mixing Complex Wavelet Transform}

The Discrete Complex Wavelet Transform (DCWT) is a complex-value of the real-valued Discrete Wavelet Transform (DWT). Unlike the 2D-DWT, the 2D-DCWT uses a complex mother wavelet where the input signals are decomposed into real and imaginary coefficients in the transform domain using a complex-valued filtering (analytic filter). Complex wavelet coefficients can be calculated using Mallat's algorithm. ${ }^{27}$

Several versions of the 2D wavelet transform have been developed, ${ }^{25}$ where the scaling and wavelet functions can be defined as

$$
\begin{aligned}
& \phi_{j, k}(x)=2^{\left(j_{1}+j_{2}\right) / 2} \phi\left(2^{j_{1}} x_{1}-k_{1}, 2^{j_{2}} x_{2}-k_{2}\right), \\
& \psi_{j, k}^{d}(x)=2^{\frac{\left(j_{1}+j_{2}\right)}{2}} \psi^{d}\left(2^{j_{1}} x_{1}-k_{1}, 2^{j_{2}} x_{2}-k_{2}\right),
\end{aligned}
$$

where $x=\left(x_{1}, x_{2}\right) \in \mathbb{R}^{2} j=\left(j_{1}, j_{2}\right) \in \mathbb{Z}^{2}, k=\left(k_{1}, k_{2}\right)$ $\in \mathbb{Z}^{2}$ and $d \in(H, V, D)$ denote one of three different directional-orientations: Horizontal $(H)$, Vertical $(V)$ and Diagonal $(D)$. The associated two-dimensional scaling function $\phi(x, y)$ and three wavelets $\psi^{d}(x, y)$ in three directions are given respectively by

$$
\begin{aligned}
\phi(x, y) & =\phi(x) \cdot \phi(y) \\
\psi^{H}(x, y) & =\phi(x) \cdot \psi(y) \\
\psi^{V}(x, y) & =\psi(x) \cdot \phi(y) \\
\psi^{D}(x, y) & =\psi(x) \cdot \psi(y) .
\end{aligned}
$$

As it can be observed, any function $f \in \mathcal{L}_{2}\left(\mathbb{R}^{2}\right)$ in the multiresolution context can be expressed as

$$
\begin{aligned}
f(x, y)= & \sum_{k} c_{\left(J_{0}, J_{0}\right), k} \phi_{\left(J_{0}, J_{0}\right), k}(x, y) \\
& +\sum_{j>J_{0}} \sum_{k} d_{\left(J_{0}, j\right), k} \psi_{\left(J_{0}, j\right), k}^{H}(x, y)
\end{aligned}
$$




$$
\begin{aligned}
& +\sum_{j>J_{0}} \sum_{k} d_{\left(j, J_{0}\right), k} \psi_{\left(j, J_{0}\right), k}^{V}(x, y) \\
& +\sum_{j_{1}, j_{2}>j_{0}} \sum_{k} d_{\left(j_{1}, j_{2}\right), k} \psi_{\left(j_{1}, j_{2}\right), k}^{D}(x, y) .
\end{aligned}
$$

which defines the new scale-mixing wavelet transform. The new scale-mixing approximation and detail coefficients are defined as

$A_{\left(J_{0}, J_{0}\right), k}=2^{J_{0}} \iint f(x, y) \bar{\phi}\left(2^{J_{0}} x-k_{1}, 2^{J_{0}} y-k_{2}\right) d x d y$,

$d_{\left(J_{0}, j\right), k}^{H}=2^{\left(J_{0}+j\right) / 2} \iint f(x, y) \overline{\psi^{H}}\left(2^{J_{0}} x-k_{1}, 2^{j} y-k_{2}\right) d x d y$,

$d_{\left(j, J_{0}\right), k}^{H}=2^{\left(j+J_{0}\right) / 2} \iint f(x, y) \overline{\psi^{V}}\left(2^{j} x-k_{1}, 2^{J_{0}} y-k_{2}\right) d x d y$,

$d_{\left(j_{1}, j_{2}\right), k}^{D}=2^{\left(j_{1}+j_{2}\right) / 2} \iint f(x, y) \overline{\psi^{D}}\left(2^{j_{1}} x-k_{1}, 2^{j_{2}} y-k_{2}\right) d x d y$,

where $j=\left(j_{1}, j_{2}\right)$, and $J_{0}$ is the smaller value of the level $j$ which can be defined as:

$$
j=\left(j_{1}, j_{2}\right), \quad j_{1}, j_{2}=j_{0}, \ldots, \log _{2}(n)-1 .
$$

$J_{0}$ denotes the lowest resolution of the transform, and larger values of $j$ correspond to higher resolutions, $\bar{\phi}$ and $\bar{\psi}^{d}$ are the complex conjugates of $\phi$ and $\psi^{d}$, respectively.

The complex scale mixing detail coefficients in Eq. (5) are related to the original image pixels through a matrix equation as in the conventional wavelet transform. The complex scale mixing coefficients are given by

$$
G=W F W^{T},
$$

where $W$ is a $2^{n} \times 2^{n}$ complex scaling matrix and $W^{T}$ denotes its Hermitian transpose, and $F$ is the $2^{n} \times 2^{n}$ input image (matrix) to be analyzed. The resulting matrix in Eq. (6) is called the scale-mixing wavelet transform of the matrix $F$ (the input image), which represents a 2D-implementation of Eq. (4) for the signal $f(x, y)$ sampled in a matrix form $(F)$.

Figure 1 illustrates the multiresolution 2D wavelet decomposition with four levels using a conventional wavelet transform and the SMCWT. By observing Fig. 1(b), it is seen that several hierarchies of subbands can be identified at each level. Similarly to the conventional 2D wavelet transform, the input images are partitioned into four subbands at the first level of SMCWT, including one approximation subband and three detail subbands which contain abundant detail information oriented along the horizontal, vertical and diagonal directions. The approximation subband generated after one level of decomposition represents the input of the next level of decomposition. The process of decomposition is iteratively repeated using Eq. (6) for $l$ levels, applied only to the approximation subband of the previous level of decomposition. We note that for a given level $l$, the horizontal and vertical detail subbands are divided into multiple matrices. Therefore, if the decomposition level is $l$, then the horizontal and vertical detail subbands result in $l$ matrices. The diagonal detail subband and the approximation subband are generated as in the conventional $2 \mathrm{D}$ wavelet decomposition at all $l$ levels.

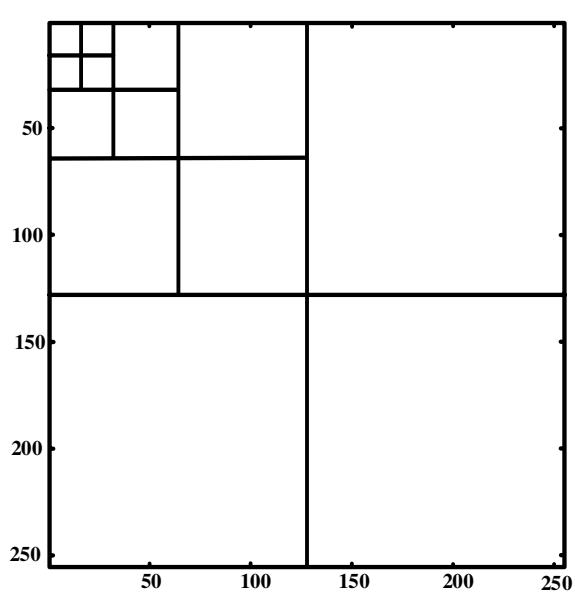

(a)

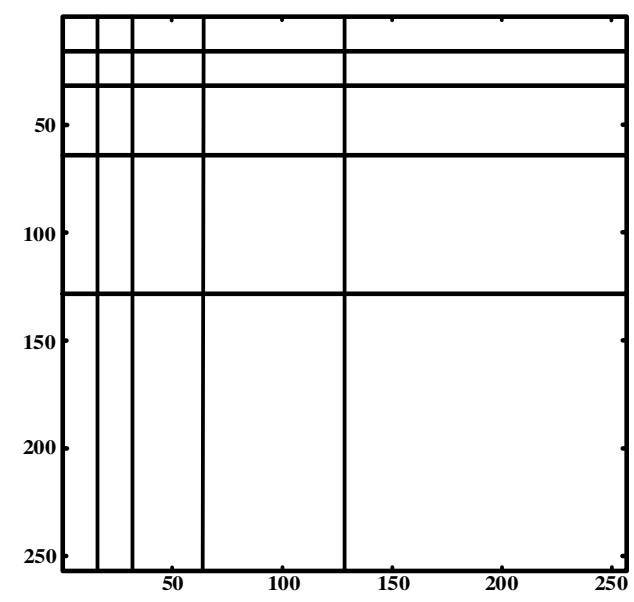

(b)

Fig. 1. Multiresolution 2D wavelet decomposition (4-levels). (a) Conventional 2D wavelet decomposition, (b) 2D Scale-mixing wavelet decomposition. 
The construction of the wavelet matrix $W$ is computationally fast. Since $W$ is orthogonal, the inverse transform is straightforward. Hence, the original image can be reconstructed from the wavelet decomposition structure as follows:

$$
F=W^{T} G W .
$$

The inherent proprieties 2D-SMCWT (symmetry, compact support, orthogonality, etc.) make it suitable for image fusion applications and other dimension-reduction applications like denoising and compression. For these reasons, we have used the 2D-SMCWT in the proposed fusion algorithm.

\section{The Proposed Fusion Method}

As noted above, the main step in the wavelet-based image fusion techniques relies on the wavelet coefficients combination, and the main goal is to reconstruct an image with all useful information contained in the wavelet coefficients of the decomposed input images. In the present work, we have processed the approximation and the detail wavelet coefficients separately using different fusion rules due primarily to the different characteristics of the wavelet coefficients. The approximation coefficients are selected by the Max-PCA fusion rule, while the detail coefficients are fused using a Bayesian MAP estimator. The overall schematic diagram illustrating our proposed fusion method is shown in Fig. 2.

It is well known that there exist two forms of representations of complex wavelet coefficients, namely, the rectangular and polar forms. In the present work, we use the polar representation since it has better shift-invariance property than the individual real and imaginary components of the rectangular representation. Both the magnitude and phase components are needed to reconstruct the fused image. In order to keep the proposed fusion method computationally efficient, only the magnitude components of the complex wavelet coefficients are considered during the estimation process, while maintaining the phase of the wavelet coefficients unchanged. Hence, the proposed fusion method is efficient and less time consuming.

\subsection{Fusion of detail coefficients}

Since an image can be considered as a $2 \mathrm{D}$ random signal, the detail coefficients obtained by the

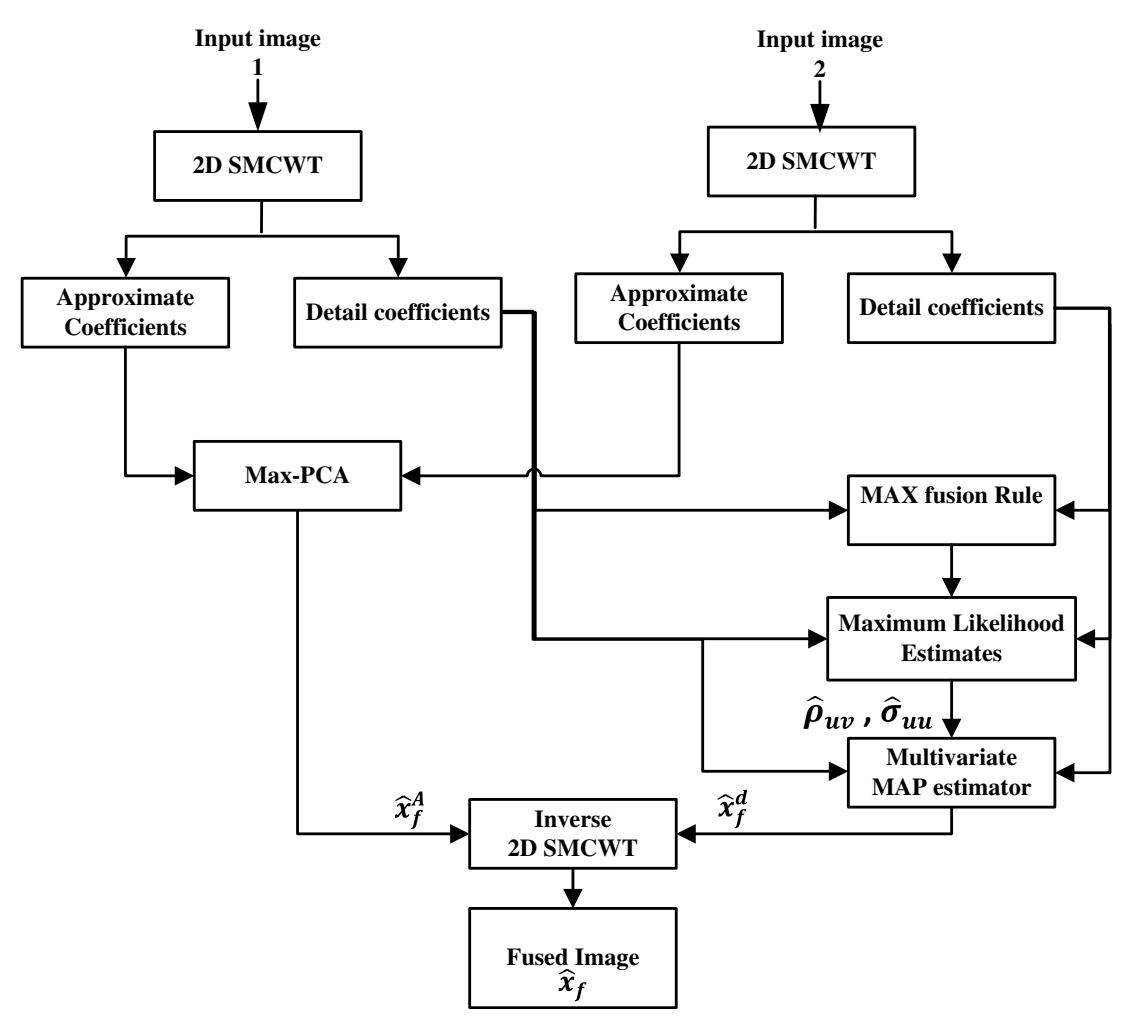

Fig. 2. Schematic diagram of the proposed fusion algorithm. 
wavelet decomposition of the input images are also random variables.

Let $X_{1}^{d}(K)$ and $X_{2}^{d}(K)$ denote the 2D-SMCWT coefficients of the input images having labels 1 and 2 , respectively, at the spatial location $K\left(k_{1}, k_{2}\right)$, of a given subband $d$ where $d \in(H, V, D)$. Also, let $x_{1}^{d}(K)$ and $x_{2}^{d}(K)$ represent the observed values of the random variables $X_{1}^{d}(K)$ and $X_{2}^{d}(K)$, respectively. The 2D-SMCWT coefficients of the input images in each subband are spatially nonstationary. Therefore, the random variables of the 2D-SMCWT coefficients are index dependent. The use of a prior information regarding random variables $X_{1}^{d}(K)$ and $X_{2}^{d}(K)$ may considerably enhance the obtained fused image. For these reasons, we have designed an efficient fusion method under a statistical estimation formulation. We have used the Maximum a Posteriori (MAP) estimation technique to obtain an estimate of $X_{f}^{d}(K)$ that denotes the random variable for the 2D-SMCWT coefficients of the fused image at the spatial location $(K)$. The mode of the posterior density $p_{X_{f}^{d}(K) \mid X_{1}^{d}(K), X_{2}^{d}(K)}$ is used as an estimate of $X_{f}^{d}(K)$. Thus, for a given decomposition level $l$, the fused coefficients of the detail subbands are given by

$$
\begin{aligned}
\hat{x}_{f}^{d}(K)= & \underset{x_{d}^{f}(k)}{\arg \max } p_{X_{f}^{d}(K) \mid X_{1}^{d}(K), X_{2}^{d}(K)} \\
& \times\left(x_{f}^{d}(K) \mid x_{1}^{d}(K), x_{2}^{d}(K)\right) \\
= & \underset{x_{d}^{f}(k)}{\arg \max } \frac{p_{X_{1}^{d}(K), X_{2}^{d}(K), X_{f}^{d}(K)}\left(x_{1}^{d}(K), x_{2}^{d}(K), x_{f}^{d}(K)\right)}{p_{X_{1}^{d}(K), X_{2}^{d}(K)}\left(x_{1}^{d}(K), x_{2}^{d}(K)\right)} \\
\propto & \underset{x_{d}^{f}(k)}{\arg \max } p_{X_{1}^{d}(K), X_{2}^{d}(K), X_{f}^{d}(K)}\left(x_{1}^{d}(K), x_{2}^{d}(K), x_{f}^{d}(K)\right),
\end{aligned}
$$

where $p_{X_{1}^{d}(K), X_{2}^{d}(K), X_{f}^{d}(K)}\left(x_{1}^{d}(K), x_{2}^{d}(K), x_{f}^{d}(K)\right)$ is the joint probability density likelihood function of $X_{1}^{d}(K), X_{2}^{d}(K), X_{f}^{d}(K)$.
The multimodal input images to be fused are captured from the same scene with different sensors. These input images should be correlated with each other in the pixel domain. Intuitively, this correlation leads to a significant level of correlation between the local neighboring 2D-SMCWT coefficients of a given subband of two input images because 2D-SMCWT is a linear transformation. ${ }^{28}$ Thus, a joint estimation of these coefficients is required to obtain an enhanced fused image. The multivariate PDF of the local neighboring wavelet coefficients of two input images has an important role for such joint estimation. Furthermore, the multivariate $\mathrm{PDF}$ is preferable since it provides a better fit to the data. Using Mardia's test for multivariate normality, ${ }^{29,30}$ the locally i.i.d zero-mean bivariate Gaussian PDF suits very well as a joint probabilistic model of the random variables $X_{1}^{d}(K)$ and $X_{2}^{d}(K)$. Since the 2D-SMCWT coefficients of the fused image are obtained from a linear transformation on the coefficients of the input images, then $X_{f}^{d}(K)$ should correlate with $X_{1}^{d}(K)$ and $X_{2}^{d}(K)$. Therefore, a suitable joint PDF of $X_{1}^{d}(K)$, $X_{2}^{d}(K)$ and $X_{f}^{d}(K)$ will be the trivariate Gaussian $\mathrm{PDF}$ given by ${ }^{31}$

$$
\begin{aligned}
p_{X_{1}, X_{2}, X_{f}}\left(x_{1}, x_{2}, x_{f}\right)= & p_{X}(x)=\frac{1}{2 \pi^{\frac{3}{2}} \sqrt{|\boldsymbol{V}|}} \\
\cdot & \exp \left\{-\frac{1}{2}\left[\left(\boldsymbol{x}^{T}-\boldsymbol{m}^{T}\right)\right.\right. \\
& \left.\left.\times \boldsymbol{V}^{-1}(\boldsymbol{x}-\boldsymbol{m})\right]\right\},
\end{aligned}
$$

where $x=\left[\begin{array}{lll}x_{1} & x_{2} & x_{f}\end{array}\right]^{T}$ represents the observed samples of the random vector $X=\left[\begin{array}{lll}X_{1} & X_{2} & X_{f}\end{array}\right]^{T}$ with $\boldsymbol{m}=\left[\begin{array}{lll}m_{1} & m_{2} & m_{f}\end{array}\right]^{T}$ with the covariance matrix

$$
\begin{aligned}
& \boldsymbol{V}=\left[\begin{array}{ccc}
E\left[\left(X_{1}-m_{1}\right)\left(X_{1}-m_{1}\right)\right] & E\left[\left(X_{1}-m_{1}\right)\left(X_{2}-m_{2}\right)\right] & E\left[\left(X_{1}-m_{1}\right)\left(X_{f}-m_{f}\right)\right] \\
E\left[\left(X_{2}-m_{2}\right)\left(X_{1}-m_{1}\right)\right] & E\left[\left(X_{2}-m_{2}\right)\left(X_{2}-m_{2}\right)\right] & E\left[\left(X_{2}-m_{2}\right)\left(X_{f}-m_{f}\right)\right] \\
E\left[\left(X_{f}-m_{f}\right)\left(X_{1}-m_{1}\right)\right] & E\left[\left(X_{f}-m_{f}\right)\left(X_{2}-m_{2}\right)\right] & E\left[\left(X_{f}-m_{f}\right)\left(X_{f}-m_{f}\right)\right]
\end{array}\right], \\
& \boldsymbol{V}=\left[\begin{array}{ccc}
\sigma_{1}^{2} & \rho_{12} \sigma_{1} \sigma_{2} & \rho_{1 f} \sigma_{1} \sigma_{f} \\
\rho_{21} \sigma_{2} \sigma_{1} & \sigma_{2}^{2} & \rho_{2 f} \sigma_{2} \sigma_{f} \\
\rho_{f 1} \sigma_{1} \sigma_{f} & \rho_{f 2} \sigma_{f} \sigma_{2} & \sigma_{f}^{2}
\end{array}\right],
\end{aligned}
$$

where $\left\{\sigma_{f}, \sigma_{1}, \sigma_{2}\right\}$ and $\left\{\rho_{i j}\left(-1 \leq \rho_{i j} \leq 1\right), i, j=1,2, f\right.$, $i \neq j\}$ are the variance and the correlation coefficients, respectively. These parameters are estimated using the local neighboring 2D-SMCWT coefficients. 
For a case of centered random variables, i.e., $m_{1}=m_{2}=m_{f}=0$, Eq. (9) becomes

$$
\begin{aligned}
p_{X_{1}, X_{2}, X_{f}}\left(x_{1}, x_{2,}, x_{f}\right)= & p_{X}(x)=\frac{1}{2 \pi^{\frac{3}{2}} \sqrt{|V|}} \\
& \cdot \exp \left\{-\frac{1}{2}\left[\boldsymbol{x}^{T} \boldsymbol{V}^{-1} \boldsymbol{x}\right]\right\},
\end{aligned}
$$

where $\quad|\boldsymbol{V}|=\sigma_{1}^{2} \sigma_{2}^{2} \sigma_{f}^{2}\left(1-\rho_{2 f}^{2}\right)-\rho_{12} \sigma_{1}^{2} \sigma_{2}^{2} \sigma_{f}^{2}\left(\rho_{21}-\right.$ $\left.\rho_{2 f} \rho_{f 1}\right)+\rho_{1 f} \sigma_{1}^{2} \sigma_{2}^{2} \sigma_{f}^{2}\left(\rho_{21} \rho_{f 2}-\rho_{f 1}\right)$ the inverse of the covariance matrix is

$$
\boldsymbol{V}^{-1}=\frac{1}{|\boldsymbol{V}|}\left[C_{i j}\right], \quad i, j=1,2, f,
$$

where: $\left[C_{i j}\right]=[\text { cofactormatrix }]^{T}$. We define $\boldsymbol{R}=$ $\boldsymbol{x}^{T} \boldsymbol{V}^{-1} \boldsymbol{x}$, which can be written as

$$
\begin{aligned}
\boldsymbol{R}= & \frac{1}{|\boldsymbol{V}|}\left[\begin{array}{lll}
x_{1} & x_{2} & x_{f}
\end{array}\right]\left[\begin{array}{lll}
C_{11} & C_{12} & C_{13} \\
C_{21} & C_{22} & C_{23} \\
C_{31} & C_{32} & C_{33}
\end{array}\right]\left[\begin{array}{l}
x_{1} \\
x_{2} \\
x_{f}
\end{array}\right] \\
= & \frac{1}{|\boldsymbol{V}|}\left[\sum_{m=1}^{3} x_{m} C_{m 1} \sum_{m=1}^{3} x_{m} C_{m 2} \sum_{m=1}^{3} x_{m} C_{m 3}\right]\left[\begin{array}{l}
x_{1} \\
x_{2} \\
x_{f}
\end{array}\right] \\
= & \frac{1}{|\boldsymbol{V}|}\left[\sum_{m=1}^{2} x_{m} x_{1} C_{m 1}+x_{f} x_{1} C_{31}+\sum_{m=1}^{2} x_{m} x_{2} C_{m 2}\right. \\
& \left.+x_{f} x_{2} C_{32}+\sum_{m=1}^{2} x_{m} x_{f} C_{m 3}+x_{f}^{2} C_{33}\right] .
\end{aligned}
$$

The MAP estimator $\hat{x}_{f}^{d}(K)$ is obtained by solving the equation given by

$\frac{\partial}{\partial x_{f}}\left[\ln p_{X_{f}^{d}(K) \mid X_{1}^{d}(K), X_{2}^{d}(K)}\left(x_{f}^{d}(K) \mid x_{1}^{d}(K), x_{2}^{d}(K)\right)\right]=0$.

which is equivalent to solving the likelihood equation

$$
\frac{\partial}{\partial x_{f}}\left[\ln p_{X_{1}, X_{2}, X_{f}}\left(x_{1}, x_{2}, x_{f}\right)\right]=\frac{\partial}{\partial x_{f}}\left[\ln p_{X}(x)\right]=0 .
$$

which becomes

$$
x_{1} C_{31}+x_{2} C_{32}+2 x_{f} C_{33}=\sum_{m=1}^{2} x_{m} C_{3 m}+2 x_{f} C_{33}=0 \text {. }
$$

Thus, for a given detail subband, the wavelet coefficients of the input images are fused using the MAP estimator obtained by solving the above equation:

$$
\hat{x}_{f}^{d}=-\frac{1}{2 C_{33}} \sum_{m=1}^{2} x_{m} C_{m, 3} .
$$

However, the elements of the matrix $\boldsymbol{V}$ are required to be estimated from the coefficients of the local neighborhood. We define a square-shaped local neighborhood $N_{K}$ centered at $x_{1}^{d}(K), x_{2}^{d}(K)$ and $x_{f}^{d}(K)$ for the spatial index $K=\left(k_{1}, k_{2}\right)$. In order to estimate the parameters, the detail coefficients within the local neighborhood $N(K)$ are assumed i.i.d. Thus, we assume that the joint PDF of the detail wavelet coefficients is an i.i.d. zero mean multivariate normal PDF. In this case, the variance $\left(\hat{\sigma}_{u u}\right)$ of the wavelet coefficients of the individual images within a local neighborhood, and the correlation coefficient $\left(\hat{\rho}_{u v}\right)$ of neighboring coefficients of the two images at the same spatial location are real and may be estimated using the maximum likelihood (ML) method as ${ }^{29}$

$$
\begin{aligned}
\hat{\sigma}_{u u}(K)= & \max \left(\frac{1}{M} \sum_{e \in N(K)}\left[x_{u}(e)\right]^{2}, 0\right) . \\
\hat{\rho}_{u v}(K)= & \max \left(\operatorname { m i n } \left(\frac{1}{M \hat{\sigma}_{u u}(K) \hat{\sigma}_{v v}(K)}\right.\right. \\
& \left.\left.\times \sum_{e \in N(K)} x_{v}(e) x_{u}(e), 1\right),-1\right),
\end{aligned}
$$

where $u, v \in(1,2, f),(u \neq v), M$ is the total number of coefficients in the local neighborhood $N(K)$. The estimated values of $\hat{\sigma}_{f f}(K)$ and $\hat{\sigma}_{u f}(K)$ using Eqs. (16) and (17) require an initial estimate of the fused wavelet coefficients. We have chosen the maximum selection rule to obtain this initial estimate given by

$$
\hat{x}_{f}^{d}(K)=\left\{\begin{array}{lll}
x_{1}^{d}(K) & \text { if } & \left|x_{1}^{d}(K)\right|>\left|x_{2}^{d}(K)\right| \\
x_{2}^{d}(K) & \text { if } & \left|x_{2}^{d}(K)\right|>\left|x_{1}^{d}(K)\right|
\end{array}\right\} .
$$

In order to obtain the MAP estimates for the fused coefficients of the detail subband $\left(\hat{x}_{f}^{d}\right)$, we first calculate the ML estimates of the parameters $\hat{\sigma}_{u u}$ and $\hat{\rho}_{u v}$, and then substitute these values in Eqs. (15) and (17).

\subsection{Fusion of approximation coefficients}

$\mathrm{PCA}^{32}$ is a mathematical tool that transforms a large number of correlated variables into a small 
number of uncorrelated variables called principal components. The primary goal of PCA is data reduction with a minimum loss of information. The first component contains the most representative information of the original data. In order to enhance the spatial resolution, we propose a new fusion rule (noted Max-PCA) to obtain the approximation coefficients of the fused image. Therefore, the process of fusing the approximation coefficients using the proposed Max-PCA fusion rule consists of the following steps:

- The approximation coefficients of the two input images are arranged in two column vectors.

- Compute the empirical mean along each column vector and then subtract it from the data of each column. The resulting matrix has a $2 \times n$ dimension, where $n$ is the length of each column vector.

- Find the covariance matrix $L$ of the resulting matrix in the previous step.

- Compute the eigenvectors ev and eigenvalues $e d$ of $L$ and sort them by decreasing eigenvalue. Note that both $e v$ and $e d$ are of dimension $2 \times 2$.

- Consider the first column of $e v$ which corresponds to larger eigenvalue to compute $P_{1}$ and $P_{2}$, where: $P_{1}=\frac{e v(1)}{\sum e v}$ and $P_{2}=\frac{e v(2)}{\sum e v}$.

Finally, Max-PCA fusion rule is performed to combine approximation coefficients as follows:

$$
x_{f}^{A}=\operatorname{Max}\left(P_{1}, P_{2}\right) \times\left(x_{1}^{A}+x_{2}^{A}\right) .
$$

Once the detail and approximation coefficients of the fused image have been calculated, the inverse 2D-SMCWT is applied to provide the final fused image.

We can summarize the fusion process of the wavelet coefficients as follows (see Fig. 2):

Step 1. Apply 2D-SMCWT to the input images.

Step 2. Apply the Bayesian MAP estimation to fuse 2D-SMCWT coefficients of the detail subbands by considering the statistical model as in Eq. (15).

Step 3. For approximation subbands, we apply the proposed Max-PCA fusion rule to obtain the approximate 2D-SMCWT coefficients of the fused image

Step 4. Apply the inverse 2D-SMCWT to get the fused image.

\section{Experimental Results and Performance Evaluations}

The proposed fusion method has been applied to different modalities of medical images viz. MR and CT images. We have performed a series of tests over three different groups of multimodal images of 256 $\times 256$ pixels with 256 -level grayscale. The proposed method is compared against eleven state-of-the-art image fusion methods on multimodal medical images. These methods include Morphological Pyramid (MP) transform, ${ }^{5}$ DWT, ${ }^{8}$ Dual Tree Complex Wavelet Transform (DTCWT), ${ }^{33}$ Shift Invariant Discrete Wavelet Transform with Haar Wavelets (SIDWT), ${ }^{34}$ Discrete Cosine Harmonic Wavelet (DCHWT), ${ }^{35}$ Spinning Sharp Frequency Localized Contourlet Transform (SFLCT_SML) ${ }^{36}$ and MultiScale Weighted Gradient-Based Fusion (MWGF) ${ }^{37}$ in the transform domain techniques category. The spatial domain techniques are $\mathrm{PCA},{ }^{32} \mathrm{CBF},{ }^{38}$ Bilateral Gradient-Based Sharpness Criterion (Sharp) and Guided Image Filter (GIF)-based method. ${ }^{39}$ We have used three levels of decomposition in all chosen transform domain fusion methods. All experiments were conducted using MATLAB R2009b on the same machine with Core duo CPU $2.33 \mathrm{GHz}$ PC, 4GB RAM.

\subsection{Subjective evaluation}

Figure 3 depicts the image fusion results from the aforementioned methods applied to the first that consists of two modalities: a CT image (Fig. 3(a)) illustrating the bone structures and a MR image (Fig. 3(b)) showing the soft tissue structures of the brain. The desired fused image should contain both hard and soft tissues.

By observing Fig. 3, it can easily notice that the results generated by PCA and GIF methods (Figs. 3(d) and $3(\mathrm{k})$ respectively) do not include the information contained in the first input image (Fig. 3(a)) at the same position. Fusion results of the MP (Fig. 3(e)), CBF (Fig. 3(j)) and sharp (Fig. 3(m)) fusion methods produce distortions and aberrant information that do not correspond to any input images. Moreover, we observe that the contrast of the fused images in SFLCT_SML (Fig. 3(n)) and SIDWT (Fig. 3(h)), is lower than that of the fused image obtained by the proposed method (Fig. 3(c)).

For more clarity, Fig. 4 shows enlarged zones of the results shown in Fig. 3. One can easily see that 


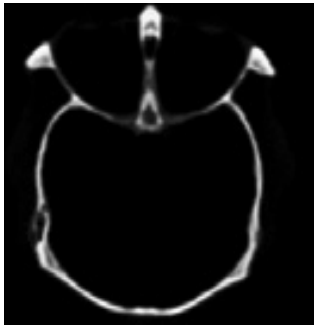

(a) CT image

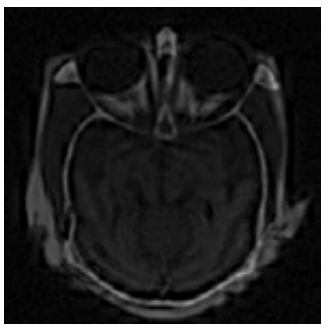

(f) DWT method

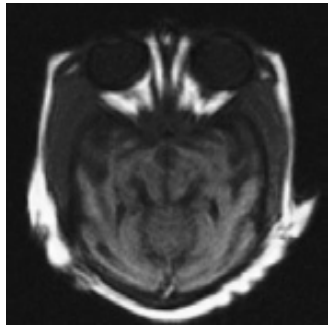

(b) MR image

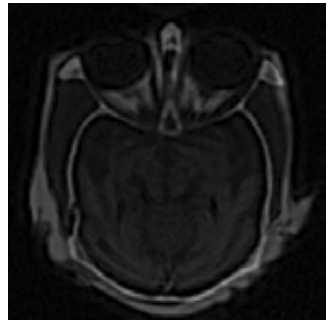

(g) DTCWT method

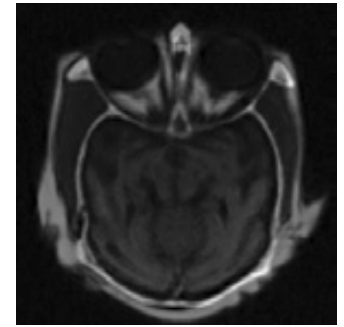

(c) Proposed method

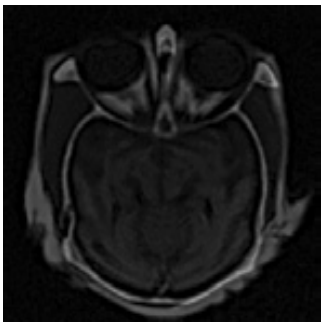

(h) SIDWT method

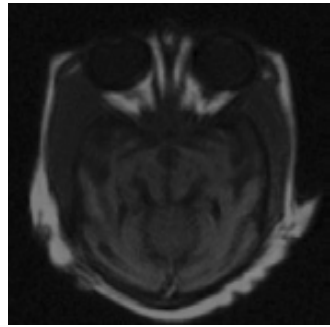

(d) PCA method

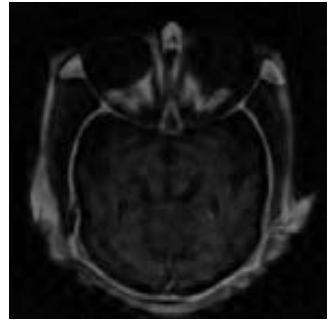

(i) DCHWT method

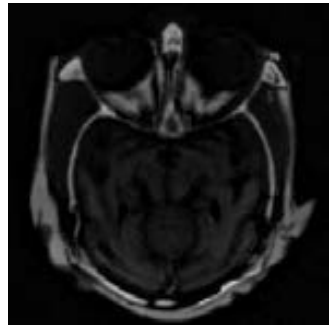

(e) MP method

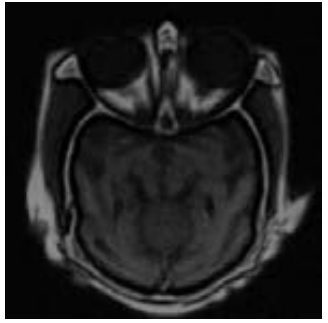

(j) CBF method

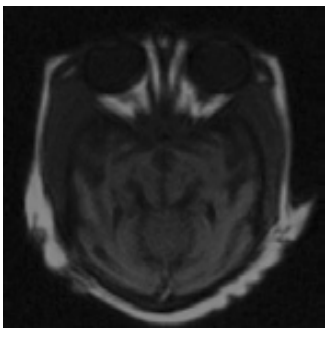

(k) GIF method

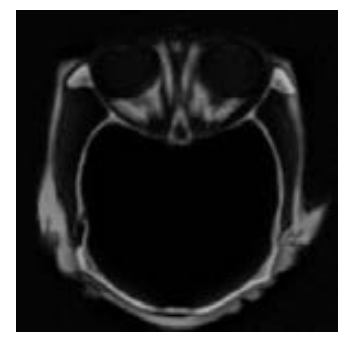

(l) MWGF method

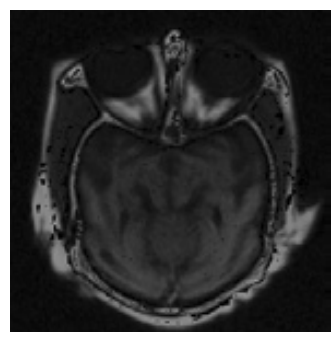

(m) Sharp method

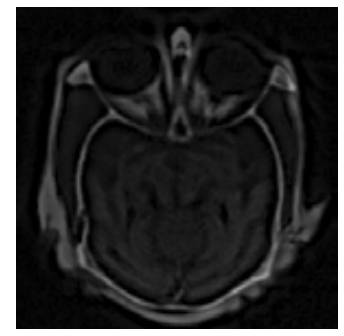

(n) SFLCT_SML method

Fig. 3. Fused images for the first group of medical images.

the proposed method preserves all relevant information from both MR and CT images and keep the highest contrast with the fewest artifacts. However, the fused images of DTCWT, SIDWT and DCHWT (Figs. 4(g)-4(i)) have a relatively low contrast with significant loss of information. The results obtained by MP-, DWT-, and CBF-based methods (Figs. 4(e)-4(f)) and Fig. 4(j)) produce several artifacts, which directly affect the visual quality of the resultant fused image. One can easily conclude from the results above that the proposed fusion method provides the best results in terms of visual quality.

Figure 5(a) presents a Magnetic Resonance Angiogram (MRA) image while Fig. 5(b) presents a T1 weighted MR image from the second group of medical images. The MRA image in Fig. 5(a) has a lower spatial resolution and contains some illness recognized as white structures (area marked by the ellipse) whereas $\mathrm{T} 1$ weighted MR image exhibits better spatial resolution and shows clearer soft tissue details. The fused images are shown in Figs. 5(c)$5(\mathrm{n})$. From these results, one can easily observe that the fused image of the proposed method is more informative and has a better visual representation. The fused images with PCA (Fig. 5(d)), CBF, GIF, MWGF and SHARP (Figs. $5(\mathrm{j})-5(\mathrm{~m})$ ) are not able to capture the information coming from the MR image and provide significant deformations. Furthermore, the proposed method provides higher contrast image compared to MP (Fig. 5(e)), DWT, DTCWT, SIDWT, DCHWT (Figs. 5(f)-5(i)) and SFLCT_SML (Fig. 5(n)) fusion methods as illustrated in Fig. 5. The fusion results for the last group of medical images ( $\mathrm{T} 1$ weighted and $\mathrm{T} 2$ weighted MR images) are shown in Fig. 6. It is easy to observe that the fused image retrieved from the proposed method has the same visual quality as the fused images obtained with the previous ones. 


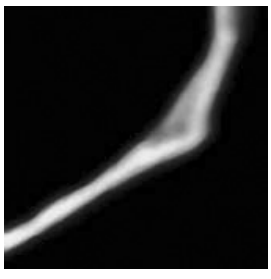

(a) CT image

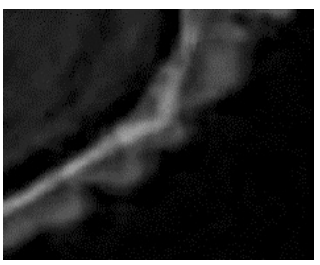

(f) DWT method

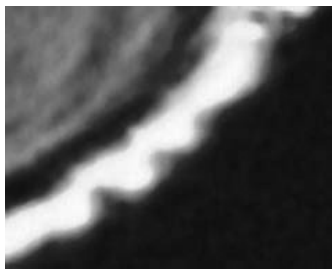

(b) MR image

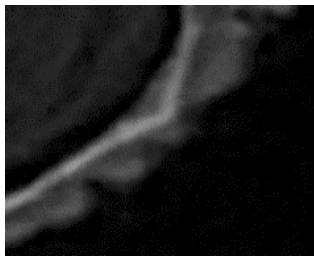

(g) DTCWT method

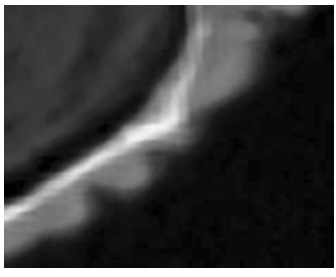

(c) Proposed method

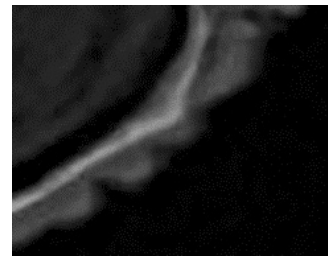

(h) SIDWT method

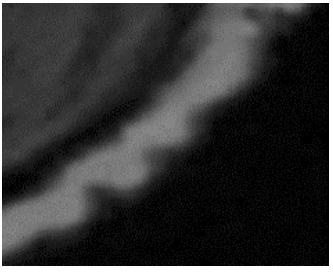

(d) PCA method

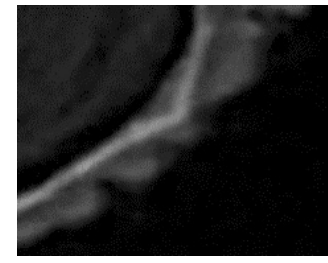

(i) DCHWT method

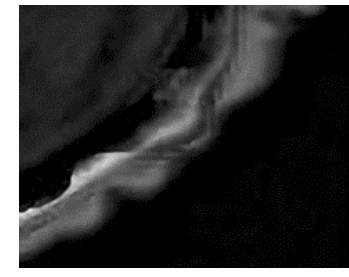

(e) MP method

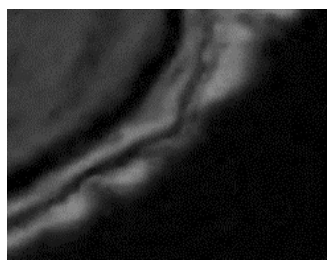

(j) CBF method

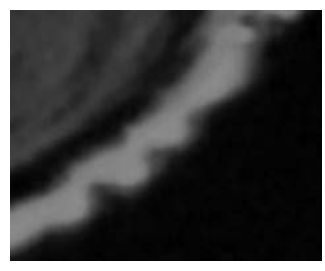

(k) GIF method

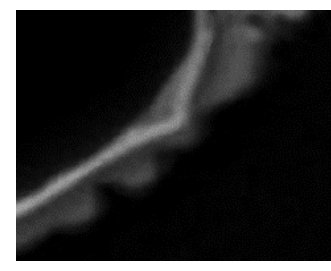

(l) MWGF method

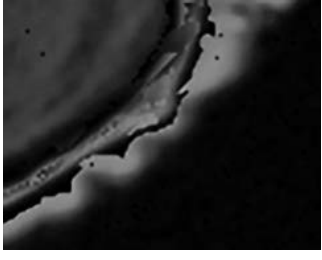

(m) Sharp method

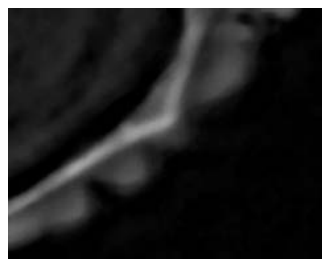

(n) SFLCT_SML method

Fig. 4. Partial enlarged regions from fused images of the first group of medical images.

\subsection{Objective evaluation}

Fusion performance evaluation is a challenging task because the ideal fused image is not available in most applications. In general, there are two categories of image fusion performance evaluation: subjective and objective assessments. ${ }^{40}$ Subjective assessments need a visual inspection by professional observers. Moreover, they are time consuming and difficult to verify. In recent years, many researchers in the field of image fusion have proposed various objective quality assessments. These metrics are automatically and easily performed using computer software. Therefore, beside the above subjective study, we perform an objective analysis of the experimental results from above using 10 recent and widely used fusion performance metrics, namely: Standard Deviation (STD), ${ }^{41}$ Entropy $(\mathrm{EN})^{41}$ Fusion Factor (FF), ${ }^{17}$ Correlation Coefficient (CC), ${ }^{42}$ Mean Structural Similarity Index Measure (MSSIM), ${ }^{43}$ Visual Information Fidelity (VIF), ${ }^{44}$ Naturalness Image Quality Evaluator (NIQE), ${ }^{45}$ Weighted Fusion Quality Index $(\mathrm{QW}),{ }^{46}$ Edge-Dependent Fusion Quality Index
$(\mathrm{QE})^{46}$ and Blind/Referenceless Image Spatial QUality Evaluator (BRISQUE) ${ }^{47}$

We have calculated the values of the fusion results for the same groups of the input images (Figs. 3 and 5-6) and tabulated them in Tables 1-3, respectively. Before discussing these tabulated values of fusion metrics, we note that better medical image fusion results should have the higher value of STD, EN, FF, CC, MSSIM, VIF, QW and QE and the lowest value of NIQE and BRISQE. From the data values of Tables 1-3, we notice that no method is better than the other according to the values of each fusion metric. For this reason, we should compare individually each method with the other ones by considering the values of all fusion metrics.

By observing the values of the fusion metrics in Table 1, we notice that the proposed method gives the best values of the objective indicators in most of the cases. The proposed method has better value of STD, EN, VIF, BRISQUE, NIQE, QW and QE, whereas GIF-based method has the best value of MSSIM and FF. This indicates that the proposed method gives better performance comparable to 


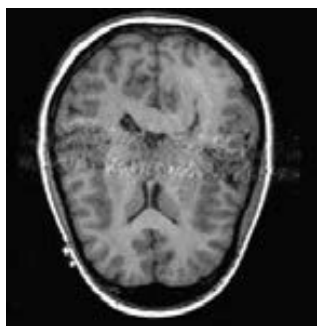

(a) T1-MR Image

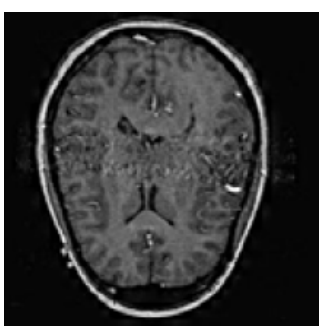

(f) DWT method

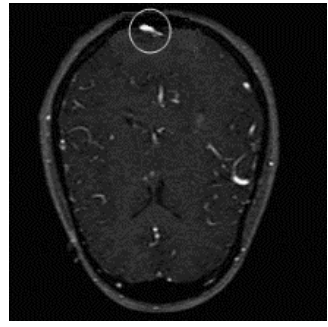

(b) MRA image

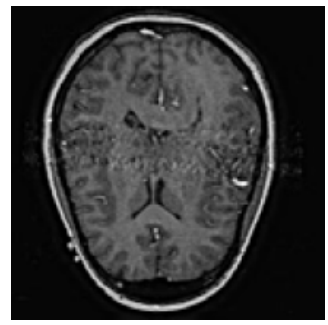

(g) DTCWT method

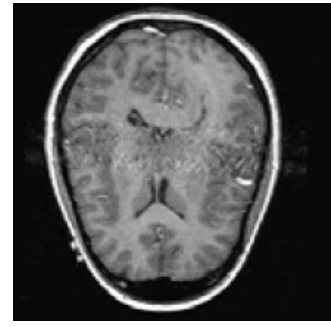

(c) Proposed method

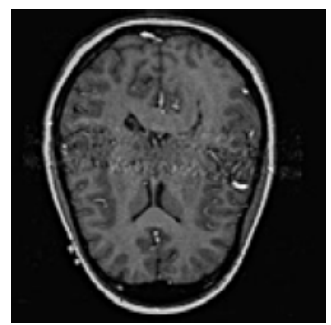

(h) SIDWT method

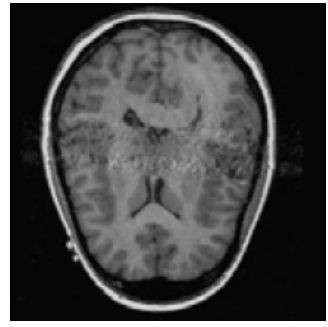

(d) PCA method

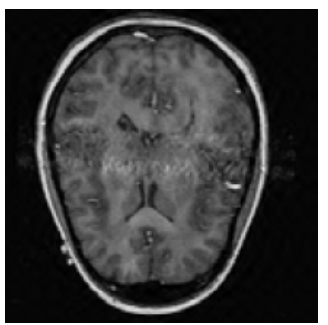

(i) DCHWT method

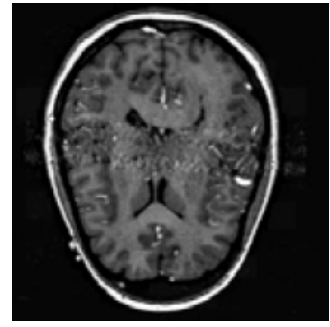

(e) MP method

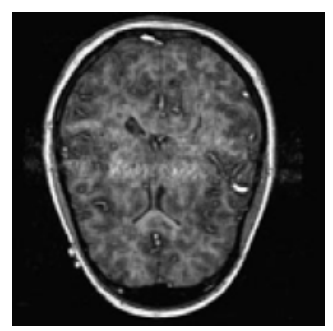

(j) CBF method

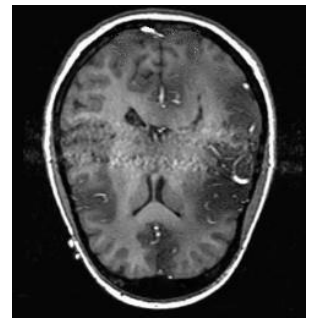

(k) GIF method

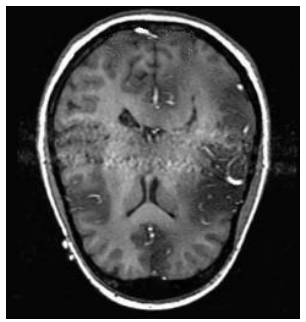

(l) MWGF method

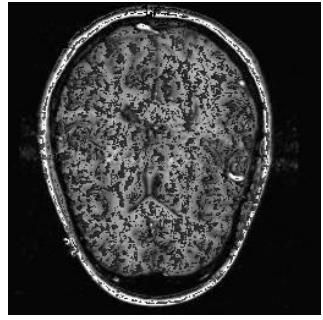

(m) Sharp method

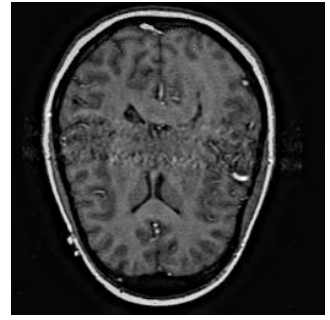

(n) SFLCT_SML method

Fig. 5. Fused images for the second group of medical images.

GIF-based method. Similarly, comparing the proposed method with other spatial domain fusion methods (PCA-, CBF- and Sharp-based methods) reveals the superiority of our method in terms of objective evaluations. Furthermore, we compare the proposed method with other transform domain fusion methods (DWT, MP, SIDWT, DCHWT and DTCWT). By examining the fusion metrics values in Table 1 again, we can easily prove that the proposed method gives better fusion results than the transform-based methods. Tables 2 and 3 show the fusion metric values from the second and the third group of input images illustrated in Figs. 5 and 6 , respectively. As observed in Table 1, the proposed method has obtained the best results in most cases regarding the other transform and spatial domain fusion methods. Although few transform methods may present better values of fusion metrics than the proposed method, a global comparison using other fusion metrics is required. Moreover, it is insufficient to analyze separately the subjective and objective evaluation of the fusion results, e.g. PCA-based method (Table 1) provides better performance than the proposed method in terms of MSSIM (0.4952) and FF (5.2780). However, it has a poor visual quality and suffers from information loss (Fig. 3(d)). Moreover, the proposed method has a higher value of BRISQUE (33.8336) (which does not imply a good fusion) than CBF-, MP- and Sharp-based methods. However, these fusion methods do not preserve all relevant information from both MR and $\mathrm{CT}$ images and produce serious distortions in the fused images.

It can be seen from Tables 2 and 3 that the proposed method has scored highly in most cases as compared to the existing methods in terms of various fusion metrics. According to Table 2, GIF and PCA-based methods have scored well in terms of MSSIM, FF and QE, even though the fused images provided by the same methods suffer from poor 


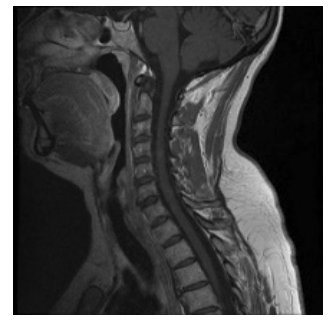

(a) T1 weighted MRI

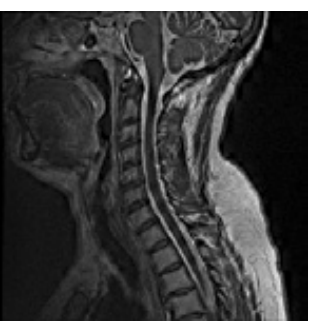

(f) DWT method

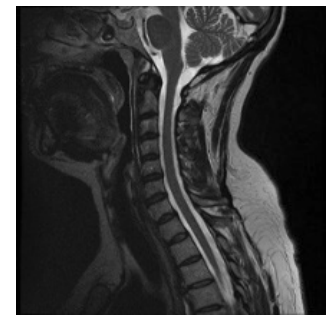

(b) T2 weighted MRI

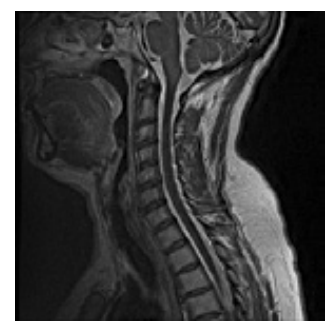

(g) DTCWT method

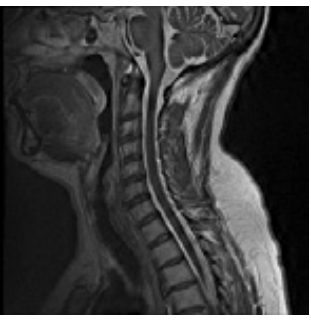

(c) Proposed method

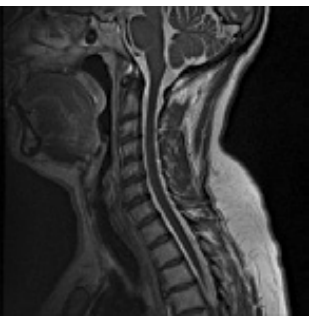

(h) SIDWT method

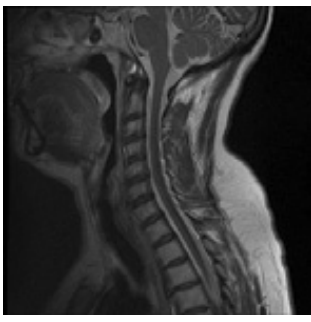

(d) PCA method

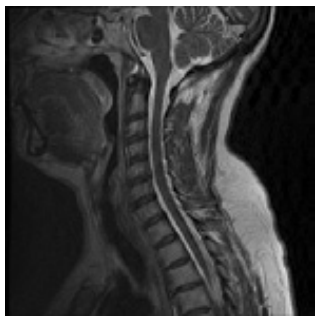

(i) DCHWT methd

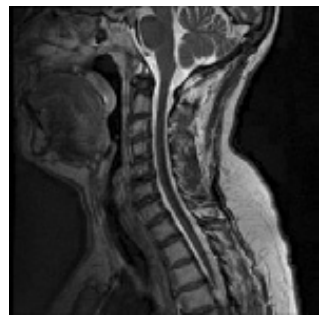

(e) MP method

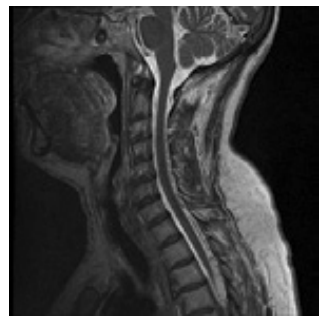

(j) CBF method

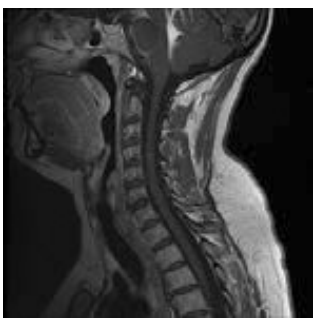

(k) GIF method

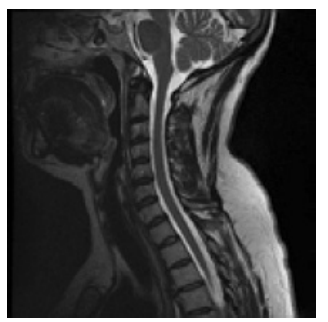

(1) MWGF method

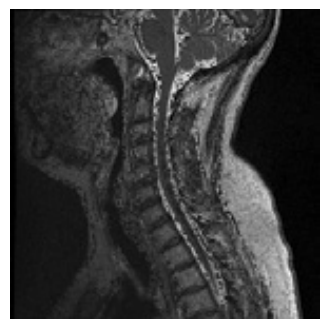

(m) Sharp method

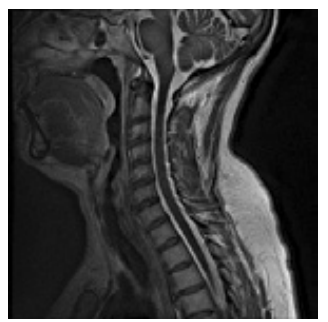

(n) SFLCT_SML method

Fig. 6. Fused images for the third group of medical images.

contrast and lose a lot of detailed information of the input images. The CBF and SFLCT_SML-based methods provide the best values of BRISTE (10.3294) and NIQE (3.4414), respectively. However, they provide a low-contrast fused image as compared to that yielded with the proposed method. Moreover, from the data values given in Table 3 , higher values of EN $(6,9946)$ and STD $(49,9128)$ are achieved with MP-based method and higher FF (7.2027) with GIF-based method. On the other

Table 1. Performance comparison for the first group of medical images.

\begin{tabular}{lcccccccccc}
\hline Methods & BRISQUE & MSSIM & STD & EN & FF & VIF & NIQE & QW & QE & CC \\
\hline Proposed method & 33.8336 & 0.3631 & 34.6367 & 5.9942 & 3.7212 & 0.6616 & 5.7048 & 0.8255 & 0.4295 & 0.7144 \\
PCA & 38.3177 & 0.4952 & 28.3806 & 5.6220 & 5.2781 & 0.2843 & 8.0305 & 0.5656 & 0.3533 & 0.5508 \\
MP & 31.5379 & 0.3341 & 29.2381 & 5.5794 & 1.9303 & 0.4380 & 6.1542 & 0.7279 & 0.3359 & 0.6558 \\
DWT & 39.2511 & 0.3222 & 22.0508 & 5.4541 & 2.1945 & 0.3313 & 7.2373 & 0.7399 & 0.3790 & 0.6953 \\
DTCWT & 36.7405 & 0.3229 & 21.4519 & 5.4505 & 2.2686 & 0.3422 & 7.7884 & 0.7362 & 0.3710 & 0.7006 \\
SIDWT & 34.8040 & 0.3296 & 23.0093 & 5.3779 & 2.4970 & 0.3895 & 6.5691 & 0.7543 & 0.4435 & 0.7041 \\
DCHWT & 41.0733 & 0.3460 & 23.3187 & 5.7434 & 1.7824 & 0.3558 & 6.5243 & 0.7450 & 0.2882 & 0.6761 \\
CBF & 33.1804 & 0.4172 & 30.6469 & 5.9157 & 2.8944 & 0.4438 & 6.7350 & 0.8414 & 0.4356 & 0.6329 \\
GIF & 36.9901 & 0.4979 & 30.0474 & 5.8723 & 4.4601 & 0.2936 & 7.8538 & 0.5302 & 0.3487 & 0.5281 \\
MWGF & 39.2431 & 0.2704 & 33.2277 & 5.0722 & 2.1105 & 0.4569 & 6.9381 & 0.7675 & 0.2995 & 0.6737 \\
SHARP & 26.5744 & 0.4244 & 30.9918 & 5.8097 & 5.5296 & 0.3501 & 11.6194 & 0.6977 & 0.3617 & 0.5817 \\
SFLCT_SML & 35.6451 & 0.3200 & 22.5327 & 5.4948 & 1.9197 & 0.3539 & 6.8717 & 0.7420 & 0.3822 & 0.6912 \\
\hline
\end{tabular}


Table 2. Performance comparison for the second group of medical images.

\begin{tabular}{|c|c|c|c|c|c|c|c|c|c|c|}
\hline Methods & BRISQUE & MSSIM & STD & EN & $\mathrm{FF}$ & VIF & NIQE & QW & $\mathrm{QE}$ & $\mathrm{CC}$ \\
\hline Propos & 11.5619 & 5190 & 66 & 6.0889 & 3.9775 & 0.8304 & 4.9656 & 0.8746 & 0.2683 & 0.9201 \\
\hline PCA & 22.7536 & 0.5313 & 56.5746 & 5.7044 & 5.2143 & 0.7448 & 5.2714 & 0.8673 & 0.3456 & 0.9016 \\
\hline MP & 25.1608 & 0.4694 & 54.5920 & 5.8559 & 3.9943 & 0.7721 & 4.1523 & 0.8735 & 0.3174 & 0.8781 \\
\hline DWT & 18.4676 & 0.4797 & 49.0719 & 5.8477 & 3.5730 & 0.5953 & 4.2959 & 0.8497 & 0.2568 & 0.9027 \\
\hline DTCWT & 20.6484 & 0.4842 & 48.3915 & 5.8934 & 3.6400 & 0.6072 & 4.2386 & 0.8490 & 0.2713 & 0.9079 \\
\hline SIDWT & 17.3609 & 0.4917 & 49.8732 & 5.6711 & 3.9088 & 0.6800 & 4.6074 & 0.8628 & 0.3015 & 0.9075 \\
\hline DCHWT & 16.4819 & 0.4745 & 50.48 & 6.2555 & 3.5623 & 0.66 & 3.5796 & 0.8628 & 0.2 & 0.9108 \\
\hline $\mathrm{CBF}$ & 10.3294 & 0.3862 & 55.5992 & 5.8597 & 3.9321 & 0.7176 & 4.7154 & 0.8 & 0.2043 & 0.8836 \\
\hline GIF & 23.7763 & 0.5344 & 64.6030 & 5.8339 & 7.2027 & 0.8516 & 5.1223 & 0.8650 & 0.4024 & 0.8838 \\
\hline MWGF & 19.0092 & 0.3925 & 61.4758 & 5.8911 & 4.4029 & 0.8192 & 4.5859 & 0.8818 & 0.3939 & 0.8860 \\
\hline SHARP & 92.1482 & 0.3842 & 58.9186 & 5.8815 & 6.4834 & 0.4754 & 18.2050 & 0.6507 & 0.0673 & 0.7757 \\
\hline SFLCT_SML & 19.7374 & 0.4809 & 50.4341 & 5.7974 & 3.5792 & 0.6818 & 3.4414 & 0.8594 & 0.2763 & 0.8962 \\
\hline
\end{tabular}

Table 3. Performance comparison for the third group of medical images.

\begin{tabular}{|c|c|c|c|c|c|c|c|c|c|c|}
\hline Methods & BRISQUE & MSSIM & STD & $\mathrm{EN}$ & $\mathrm{FF}$ & VIF & NIQE & QW & $\mathrm{QE}$ & $\mathrm{CC}$ \\
\hline Proposed method & 0.4274 & 0.6860 & 47.2334 & 6.9614 & 4.2056 & 0.6305 & 3.9784 & 0.8030 & 0.2402 & 0.8949 \\
\hline PCA & 7.3229 & 0.6922 & 41.5344 & 6.7812 & 4.7965 & 0.5575 & 4.4391 & 0.7340 & 0.2026 & 0.9089 \\
\hline MP & 3.8865 & 0.6389 & 49.9128 & 6.9946 & 4.0117 & 0.5994 & 4.2203 & 0.7762 & 0.2500 & 0.8581 \\
\hline DWT & 7.7424 & 0.6543 & 46.3339 & 6.8981 & 3.7869 & 0.5798 & 4.2588 & 0.7796 & 0.2194 & 0.8774 \\
\hline DTCWT & 4.0047 & 0.6892 & 45.3997 & 6.8894 & 3.9791 & 0.5962 & 4.2475 & 0.8040 & 0.2405 & 0.8889 \\
\hline SIDWT & 1.8425 & 0.6915 & 45.4975 & 6.8621 & 4.0902 & 0.6188 & 4.4246 & 0.8103 & 0.2831 & 0.8898 \\
\hline DCHWT & 2.7385 & 0.6874 & 44.1991 & 6.9221 & 4.2160 & 0.5612 & 4.0232 & 0.8076 & 0.2320 & 0.8973 \\
\hline $\mathrm{CBF}$ & 3.2966 & 0.6520 & 45.9108 & 6.9212 & 4.7552 & 0.5347 & 3.3304 & 0.7951 & 0.2370 & 0.8792 \\
\hline GIF & 8.4099 & 0.6111 & 46.1865 & 6.7930 & 7.9305 & 0.4371 & 4.1055 & 0.4952 & 0.2570 & 0.8360 \\
\hline MWGF & 2.8793 & 0.6107 & 48.3071 & 6.7424 & 7.0820 & 0.4524 & 4.5740 & 0.7495 & 0.3578 & 0.8430 \\
\hline SHARP & 51.7888 & 0.6495 & 46.8073 & 6.9288 & 6.4923 & 0.4826 & 11.9432 & 0.6216 & 0.1240 & 0.8310 \\
\hline SFLCT_SML & 0.1427 & 0.6806 & 45.6656 & 6.9288 & 3.8783 & 0.5793 & 3.8022 & 0.8009 & 0.2666 & 0.8831 \\
\hline
\end{tabular}

Table 4. Computational efficiency for the three groups of medical images.

\begin{tabular}{lccc}
\hline Execution time (s) & $\begin{array}{c}\text { ET for the first } \\
\text { group of images }\end{array}$ & $\begin{array}{c}\text { ET for the second } \\
\text { group of images }\end{array}$ & $\begin{array}{c}\text { ET for the third } \\
\text { group of images }\end{array}$ \\
\hline Proposed method & 0.2240 & 0.2408 & 0.1708 \\
PCA & 0.0035 & 0.0050 & 0.0087 \\
MP & 0.7903 & 1.0857 & 0.5854 \\
DWT & 0.1302 & 0.1535 & 0.0716 \\
DTCWT & 0.2706 & 0.2888 & 0.1712 \\
SIDWT & 0.4830 & 0.4962 & 0.2658 \\
DCHWT & 3.7837 & 3.8114 & 1.4386 \\
CBF & 42.4435 & 43.3482 & 17.1381 \\
GIF & 0.0562 & 0.0558 & 0.0941 \\
MWGF & 3.1829 & 3.8904 & 2.7468 \\
SHARP & 14.3441 & 14.9402 & 4.2599 \\
SFLCT_SML & 19.4733 & 21.6911 & 9.5029 \\
\hline
\end{tabular}

hand, the fused image obtained using the MP-based method has a new pattern that does not exist in any of the input images. Similar result is provided by sharp-based method, which creates artifacts in the fused image. From the above comparison, one can easily conclude that the proposed method gives better results than transform and spatial domain fusion methods. Further, the visual quality of the 
A. Bengueddoudj, Z. Messali \& V. Mosorov

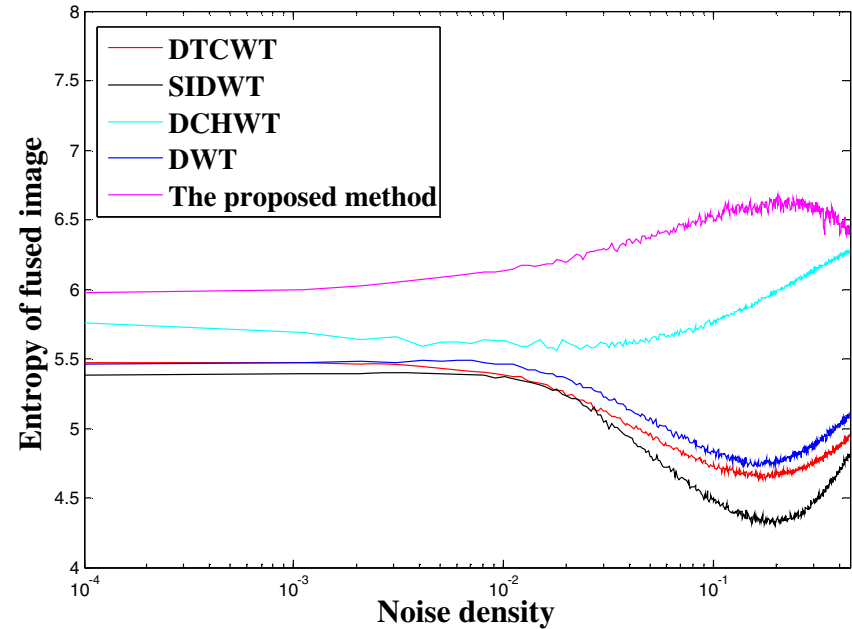

(a) Plot of EN fusion metric against salt and pepper noise

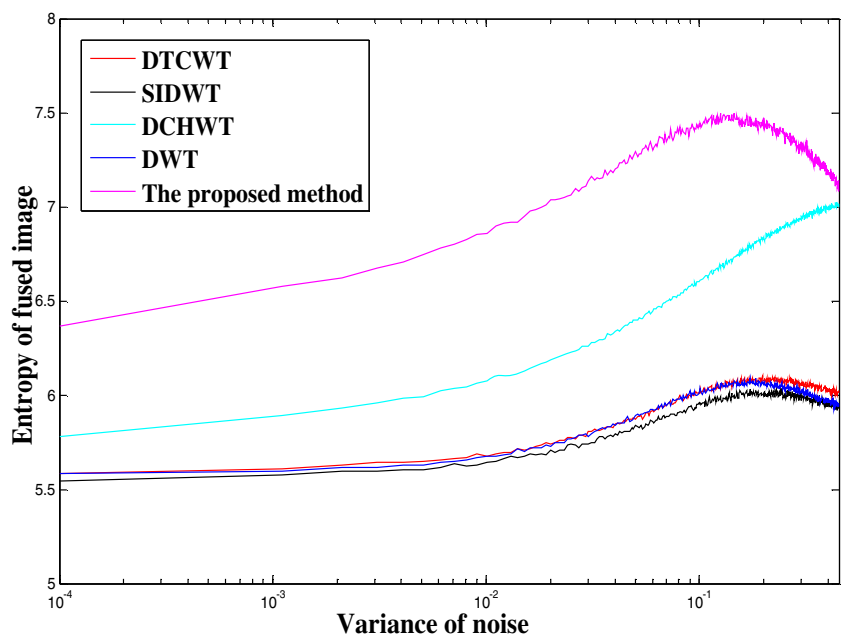

(c) Plot of EN fusion metric against zero mean Gaussian noise

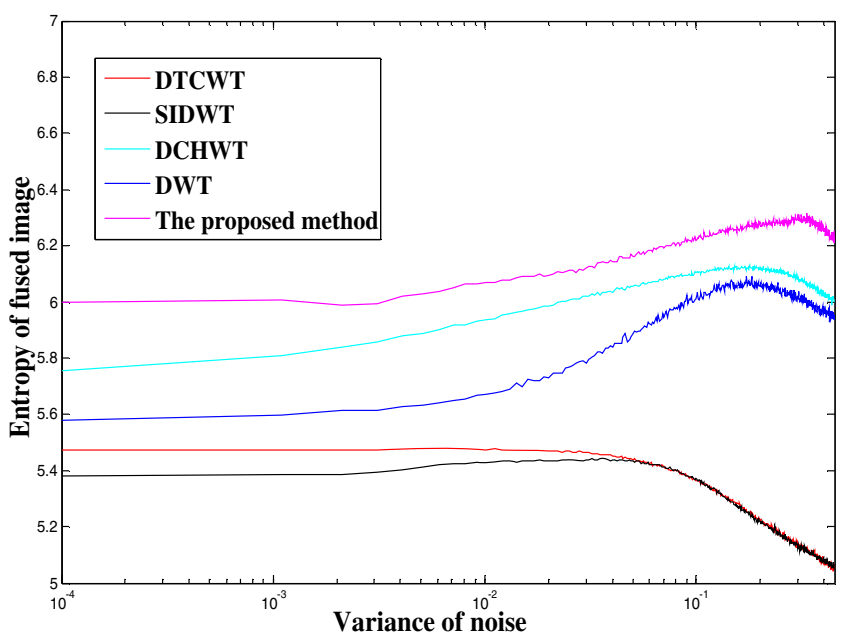

(e) Plot of EN fusion metric against speckle noise

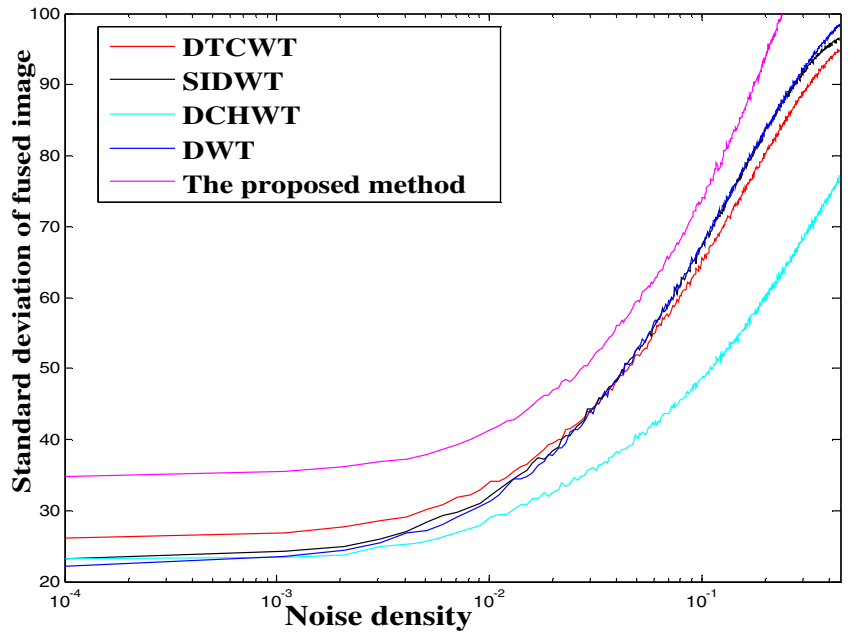

(b) Plot of STD fusion metric against salt and pepper noise

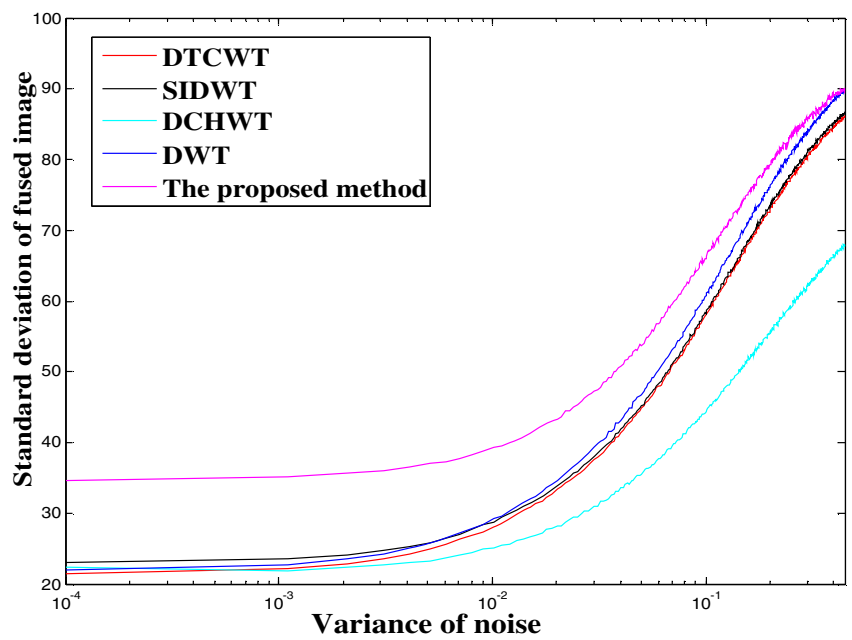

(d) Plot of STD fusion metric against zero mean Gaussian noise

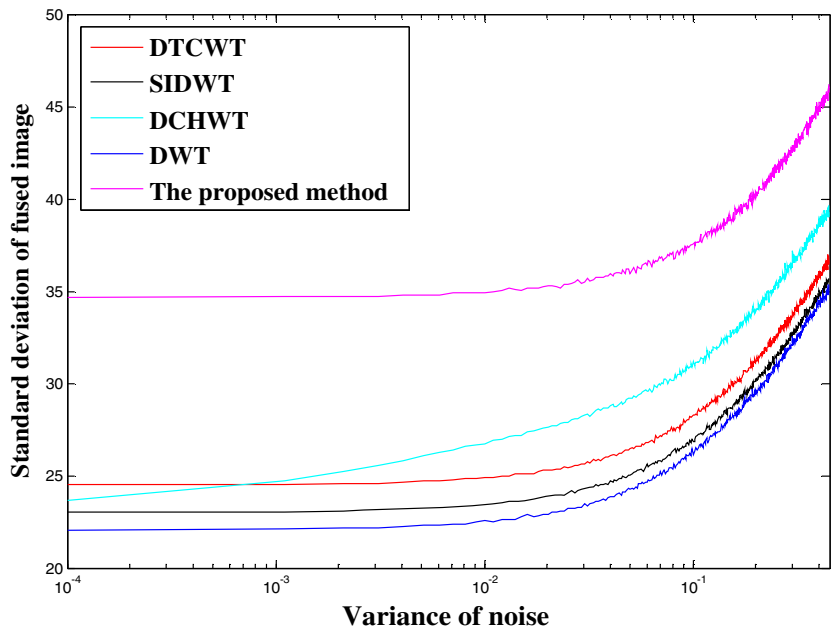

(f) Plot of STD fusion metric against speckle noise

Fig. 7. Plots of EN and STD against different types of noise. 
fused images obtained by the proposed method are in agreement with the objective evaluation based on the values of different fusion metrics over the three groups of medical images.

Execution time plays an important role in medical applications that require real time processing, therefore, we evaluate here the complexity of the proposed method compared to the considered fusion methods in this study. As shown in Table 4, the execution times (ETs) required to generate the fused image using the proposed method are $0.22 \mathrm{~s}$, $0.24 \mathrm{~s}$ and $0.17 \mathrm{~s}$ for the three groups of multimodal medical images, i.e., computational time compared to the other methods is lower. Even though, Table 4 shows that PCA- and GIF-based methods have the best computational time, these methods show poor results in terms of visual quality and quantitative evaluation as noted before. Therefore, the proposed fusion method can be considered to be highly effective since it offers a reasonable balance between computational complexity on one hand, and quantitative and visual results on the other hand.

To demonstrate the robustness of the proposed method against different types of noise (zero mean Gaussian, salt and pepper and speckle), we have performed experiments by adding different values of noise variance and noise density to input images. The experiments were carried out with different types of noise in order to evaluate the robustness of the proposed method as compared to other waveletbased fusion methods including DTCWT, DCHWT, SIDWT and DWT. A large number of tests (450 tests) are conducted through an extensive experiment in terms of Entropy and Standard Deviation. Plots of fusion metrics against different levels of Gaussian, salt and pepper and speckle noise for the first group of medical images are shown in Fig. 7. By observing plots of STD and EN in Fig. 7, it is clear that the fused image of the proposed method is not affected by noise attacks since the value of STD and EN indices are continuously increasing as the level of noise increases. Furthermore, the proposed method has the highest values of STD and EN regarding other compared fusion methods. The plots of STD against the three different types of noise using DTCWT-, SIDWT- and DWT-based methods show good fusion results. However, the plots of EN against salt and pepper noise using these methods and speckle noise using DTCWT- and SIDWT-based methods in Figs. 7(a) and 7(e) respectively, show the poor robustness against noise.

\section{Conclusion}

In this paper, we have proposed a new and efficient image fusion method based on 2D-SMCWT which is more compressive and preserves the energy. A trivariate $\mathrm{PDF}$ has been used to model the detail $2 \mathrm{D}$ SMCWT coefficients of the input images and the fused image. These coefficients are estimated using a Bayesian MAP estimation approach. We also proposed a new fusion rule named 'Max-PCA' to combine the approximation 2D-SMCWT coefficients. The proposed fusion method has been compared with several known methods through the experiments conducted on three different groups of multimodal medical images. The visual comparison shows that the proposed method has significantly better fusion performances than the other methods, as well as with respect to the standard image fusion performance measures. Furthermore, the fusion measure plots of entropy and standard deviation metrics against Gaussian, salt and pepper and speckle noise show that the proposed fusion method performs well in the presence of noise and is better than the considered wavelet domain image fusion methods.

\section{Acknowledgments}

The authors would like to sincerely thank the anonymous reviewers for their valuable comments and suggestions that helped us improve the paper.

\section{References}

1. Q. Wang, S. Li, H. Qin, A. Hao, "Robust multimodal medical image fusion via anisotropic heat diffusion guided low-rank structural analysis," Inf. Fusion 26, 103-121 (2015).

2. D. Delbeke, R. E. Coleman, M. J. Guiberteau, M. L. Brown, H. D. Royal, B. a Siegel, D. W. Townsend, L. L. Berland, J. A. Parker, G. Zubal, V. Cronin, "Procedure guideline for SPECT/CT imaging 1.0," J. Nucl. Med. 47, 1227-1234 (2006).

3. P. Ganasala, V. Kumar, "CT and MR image fusion scheme in nonsubsampled contourlet transform domain," J. Digit. Imaging 27, 407-418 (2014).

4. W. Ha, P. H. Gowda, T. A. Howell, "A review of potential image fusion methods for remote sensingbased irrigation management: Part II," Irrig. Sci. 31, 851-869 (2013).

5. N. Uniyal, S. K. Verma, "Image fusion using morphological pyramid consistency method," International Journal of Computer Applications 95, 34-38 (2014). 
6. K. Amolins, Y. Zhang, P. Dare, "Wavelet based image fusion techniques - An introduction, review and comparison," ISPRS J. Photogramm. Remote Sens. 62, 249-263 (2007).

7. H. Li, B. S. Manjunath, S. K. Mitra, "Multisensor image fusion using the wavelet transform," Graphical Models and Image Processing 57, 235-245 (1995).

8. L. Chiorean, M.-F. Vaida, Medical image fusion based on discrete wavelet transform using Java technology, Proc. ITI 2009 31st Int. Conf. Information Technology Interfaces, pp. 55-60 (2009).

9. Y. Bo, J. Zhongliang, Z. Haitao, "Review of PixelLevel Image Fusion," J. Shanghai Jiaotong Univ. (Sci.) 15, 6-12 (2010).

10. J. J. Lewis, R. J. O'Callaghan, S. G. Nikolov, D. R. Bull, N. Canagarajah, "Pixel- and region-based image fusion with complex wavelets," Inf. Fusion 8, 119-130 (2007).

11. J. Liu, P. Moulin, "Information-theoretic analysis of interscale and intrascale dependencies between image wavelet coefficients," IEEE Trans. Imag. Process. 10, 1647-1658 (2001).

12. S. Yin, L. Cao, Y. Ling, G. Jin, "Fusion of noisy infrared and visible images based on anisotropic bivariate shrinkage," Infrared Phys. Technol. 54, 13-20 (2011).

13. J. Yang, R. S. Blum, A statistical signal processing approach to image fusion using Hidden Markov Models, Multi-Sensor Image Fusion and Its Applications, pp. 256-287, Marcel Dekker/CRC (2005).

14. S. Roy, T. Howlader, S. M. M. Rahman, "Image fusion technique using multivariate statistical model for wavelet coefficients," Signal, Image Video Process. 7, 355-365 (2011).

15. S. M. Mahbubur Rahman, M. Omair Ahmad, M. N. S. Swamy, "Contrast-based fusion of noisy images using discrete wavelet transform," IET Image Process. 4, 374-384 (2010).

16. A. Loza, D. Bull, N. Canagarajah, A. Achim, "NonGaussian model-based fusion of noisy images in the wavelet domain," Comput. Vis. Image Underst. 114, 54-65 (2010).

17. R. Singh, A. Khare, "Fusion of multimodal medical images using Daubechies complex wavelet transform - A multiresolution approach," Inf. Fusion 19, 4960 (2014).

18. N. Kingsbury, "Image processing with complex wavelets," Philos. Trans. R. Soc. A Math. Phys. Eng. Sci. 357, 2543-2560 (1999).

19. M. L. Gavrilova, C. J. K. Tan, K. Saeed, N. Chaki, S. H. Shaikh, Transactions on Computational Science XXV, Vol 9030, Springer, New York (2015).

20. N. Kingsbury, "Complex wavelets for shift invariant analysis and filtering of signals," Appl. Comput. Harmon Anal. 10, 234-253 (2001).
21. A. L. Da Cunha, J. Zhou, M. N. Do, "The nonsubsampled contourlet transform: Theory, design, and applications," IEEE Trans. Image Process. 15, 3089-3101 (2006).

22. Q. Miao, C. Shi, P. Xu, M. Yang, Y. Shi, "A novel algorithm of image fusion using shearlets," Opt. Commun. 284, 1540-1547 (2011).

23. Y. Yang, Y. Que, S. Huang, P. Lin, "Multimodal sensor medical image fusion based on type-2 fuzzy logic in NSCT domain," IEEE Sens. J. 16, 3735-3745 (2016).

24. G. Bhatnagar, Q. M. J. Wu, Z. Liu, "A new contrast based multimodal medical image fusion framework," Neurocomputing 157, 143-152 (2015).

25. P. Ramírez-Cobo, K. S. Lee, A. Molini, A. Porporato, G. Katul, B. Vidakovic, "A wavelet-based spectral method for extracting self-similarity measures in time-varying two-dimensional rainfall maps," J. Time Ser. Anal. 32, 351-363 (2011).

26. N. Remenyi, O. Nicolis, G. Nason, B. Vidakovic, "Image denoising with 2D scale-mixing complex wavelet transforms," IEEE Trans. Image Process. 23, 5165-5174 (2014).

27. S. Mallat, A Wavelet Tour Signal Process, Academic Press, California (1999).

28. S. M. M. Rahman, M. O. Ahmad, M. N. S. Swamy, "Statistics of 2-D DT-CWT coefficients for a Gaussian distributed signal," IEEE Trans. Circuits Syst. I Regul. Pap. 55, 2013-2025 (2008).

29. T. Howlader, Y. P. Chaubey, "Noise reduction of cDNA microarray images using complex wavelets," IEEE Trans. Image Process. 19, 1953-1967 (2010).

30. S. Barber, G. Nason, "Real nonparametric regression using complex wavelets," J. R. Stat. Soc. Ser. B 66, 927-939 (2004).

31. M. Barkat, Signal Detection and Estimation, Artech House, London (2005).

32. V. P. S. Naidu, J. R. Raol, "Pixel-level image fusion using wavelets and principal component analysis," Def. Sci. J. 58, 338-352 (2008).

33. R. Singh, R. Srivastava, O. Prakash, A. Khare, "Multimodal medical image fusion in dual tree complex wavelet transform domain using maximum and average fusion rules," J. Med. Imag. Health Inf. 2, 168-173 (2012).

34. O. Rockinger, "Image sequence fusion using a shiftinvariant wavelet transform," Proc. Int. Conf. Image Process. 3, 288-291 (1997).

35. B. K. Shreyamsha Kumar, "Multifocus and multispectral image fusion based on pixel significance using discrete cosine harmonic wavelet transform," Signal Image Video Process. 7, 1125-1143 (2013).

36. Q. Xiao-bo, Y. Jing-wen, Y. Gui-de, "Sum-modifiedLaplacian-based Multifocus Image Fusion Method 
in Sharp Frequency Localized Contourlet Transform Domain," Opt. Precis. Eng. 17, 1203-1202 (2009)

37. Z. Zhou, S. Li, B. Wang, "Multi-scale weighted gradient-based fusion for multi-focus images," Inf. Fusion 20, 60-72 (2014).

38. B. K. Shreyamsha Kumar, "Image fusion based on pixel significance using cross bilateral filter," Signal Image Video Process. 9, 1193-1204 (2015).

39. K. Zhan, J. Teng, Q. Li, J. Shi, "A novel explicit multi-focus image fusion method," J. Inf. Hiding Multimed. Sign. Process. 6, 600-612 (2015).

40. P. Mohammadi, A. Ebrahimi-Moghadam, S. Shirani, "Subjective and objective quality assessment of image: A survey," Majlesi J. Electr. Eng. 9, 55-83 (2014).

41. B. V. Dasarathy, Multi-Sensor Image Fusion and Its Applications, CRC Press 2007.

42. J. L. Crowley, J. Martin, Experimental comparison of correlation techniques, Proc. Int. Conf. Intelligent Autonomous Systems, Karlsruhe (1995).
43. Z. Wang, E. P. Simoncelli, A. C. Bovik, "Multi-scale structural similarity for image quality assessment," IEEE Asilomar Conf. Signals, Syst. Comput. 2, 9-13 (2003).

44. H. R. Sheikh, A. C. Bovik, "Image information and visual quality," IEEE Trans. Image Process. 15, 430-444 (2006).

45. A. Mittal, R. Soundararajan, A. C. Bovik, "Making a 'completely blind' image quality analyzer," IEEE Signal Process Lett. 20, 209-212 (2013).

46. G. Piella, H. Heijmans, A new quality metric for image fusion, Proc. Int. Conf. Image Processing, pp. 173-176 (2003).

47. A. Mittal, A. K. Moorthy, A. C. Bovik, "No-reference image quality assessment in the spatial domain," IEEE Trans. Image Process. 21, 4695-4708 (2012). 